\title{
Miniaturized Thin Soft Surface Structure Using Metallic Strips with Ledge Edges for Antenna Applications
}

\author{
Said A. Abushamleh ${ }^{1, *}$, Hussain Al-Rizzo ${ }^{1}$, \\ Ahmed A. Kishk ${ }^{2}$, Ayman Abbosh ${ }^{1}$, and Haider Khaleel ${ }^{3}$
}

\begin{abstract}
A new thin electromagnetic soft surface of strips in which ledge edges are used to reduce the strip period width and in turns a miniaturized structure is achieved. The surface is tested to reduce the mutual coupling between microstrip patches separated by a half wavelength in free space (center-tocenter). A $20 \%$ relative bandgap bandwidth is achieved. The measurements revealed good agreement with the simulated results.
\end{abstract}

\section{INTRODUCTION}

Electromagnetic soft surfaces are realized by corrugated conductors with a $\lambda_{\mathrm{g}} / 4$ depth or narrow printed conducting strips over a grounded dielectric substrate of thickness $\lambda_{\mathrm{g}} / 4$ where $\lambda_{\mathrm{g}}$ is the guided wavelength [1-4]. The strips can be grounded through conducting vias. Strip-type soft surfaces are simple, easy to design and fabricate compared with corrugated type [5].

The soft boundary suppresses waves propagating in the direction transverse to the corrugations or the strips for both TE and TM polarizations. The definition and realization of such surfaces are treated in detail in [1-4]. The soft surface is polarization independent, but propagation direction dependent. The new soft surface structure presented in this paper is one-dimensional suppression structure. For thin grounded dielectric substrates, the periodic strips of width, $w$, approximately $\lambda_{\mathrm{g}} / 2$ are grounded by centered vias as shown in Fig. 1 . The vias are periodic along the strips with period $p_{v}$ and the strips are separated by a gap of width $g$.

In [5-9], the effects of different parameters on the conventional planar soft surfaces are introduced along with applications of soft surfaces in GPS antennas, mutual coupling and back radiation reductions in patch antennas. In [5] different soft surfaces consisting of triangularly jagged strips were introduced.

Compared with the mushroom-type EBG structure [10], planar soft surfaces have smaller unit cell size; the unit cell size is calculated in terms of frequency at which their bandgaps appear [5]. However, EBG/HIS offers more degrees of freedom in controlling wave propagation, scattering from all directions and wide angles of incidence compared with soft surfaces [11]. The unit cell size is defined along the direction of soft, $P_{s}$, as shown in Fig. 1. In [11] the bandgap of different soft surfaces is studied based on the dispersion diagram. The corrugated and strip-loaded soft surfaces are characterized in a similar way as EBG structures. It should be pointed out that the strip width can be reduced if the vias are placed at the edge of the strip (lateral via position).

The mutual coupling and back radiation reduction for patch antennas using planar soft surfaces [5,9], and $[12,13]$ are achieved. However, in [5] the half wavelength (in free space) separation between the centers of the two patches is not reachable because of the large cell size.

Received 26 September 2013, Accepted 11 December 2013, Scheduled 13 December 2013

* Corresponding author: Said A. Abushamleh (saabushamleh@ualr.edu).

1 Department of Systems Engineering, University of Arkansas at Little Rock, 2801 S. University Ave., Little Rock, AR 72204, USA.

2 Department of Electrical \& Computer Engineering, Concordia University, 1515 St. Catherine West, SEV005.139, Montreal, Quebec

H3G 2W1, Canada. ${ }^{3}$ Department of Engineering Science, Sonoma State University, Salazar Hall 2004, 1801 East Cotati Ave.,

Rohnert Park, CA 94928, USA. 


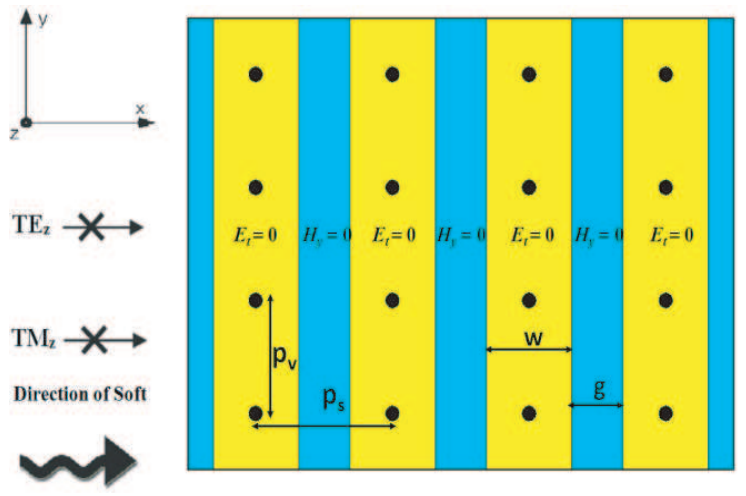

Figure 1. Conventional planar soft surface printed on a grounded dielectric slab.

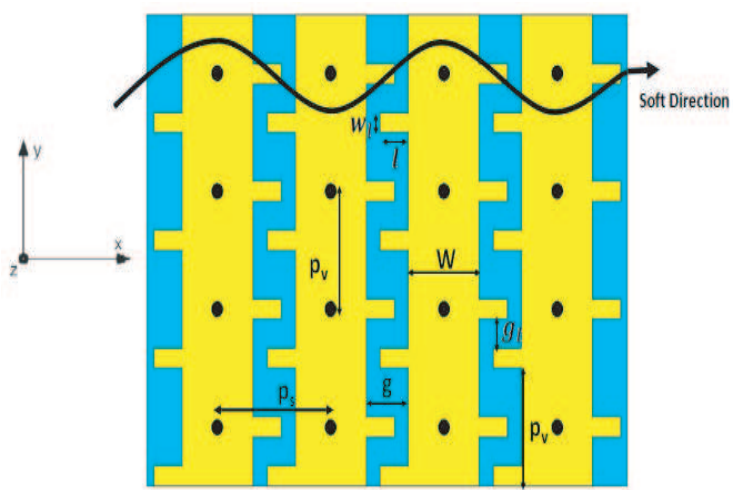

Figure 2. Soft surface structure composed of four metallic strips $(N=4)$ of interleaved ledges.

Here, we propose a planar soft surface with interleaved ledges as shown in Fig. 2. The structure is compact and easily inserted between closely separated microstrip patches and in turn the mutual coupling can be reduced between two patch antennas with center to center distance of half wavelength without affecting their radiation patterns. Such structure is more successful than other shapes such as the fork-like-EBG structure [14] where the distance between the two patches was $0.76 \lambda_{0}$, where $\lambda_{0}$ is the free space wavelength at the resonant frequency of the antenna. Finally, up to $20 \%$ relative bandgap bandwidth is achieved with the proposed structure.

\section{NUMERICAL PARAMETRIC STUDY}

In this section, we present the study on different parameters in the design to infer the effect of each parameter. Vertical polarization is assumed and finite structures are considered. These parameters determine the filtering level and the bandgap locations.

The structure shown in Fig. 2 is composed of a finite number of metallic strips on a grounded dielectric slab (Rogers RO3003 with a relative permittivity $\varepsilon_{r}=3, \tan \delta=0.0013$, and thickness $=1.524 \mathrm{~mm}$ ). The metal strips and ground plane are connected by metallic vias. CST Microwave Studio is used to calculate the $S$-parameters. The model in Fig. 2 was simulated in CST using two waveguide ports facing each other on the direction of soft ( $x$-direction) as shown in Fig. 3. Magnetic boundary conditions are used on $y_{\min }$ and $y_{\max }$, whereas Electric boundary conditions are used on $z_{\min }$ and $z_{\max }$. In the present study only the first bandgap is considered. The parameters are: Number of strips $N$, period between vias $p_{v}$, strip width $w$, ledge length $l$, ledge width $w_{l}$, vertical gap between interleaved ledges $g_{l}$ and position of vias within the strip width. Using interleaved ledges does not affect the actual gap between metallic strips. A larger unit cell size is required for the Face-to-Face ledges introduced in [15] since the actual gap between strips needs to be extended for the same length $l$.

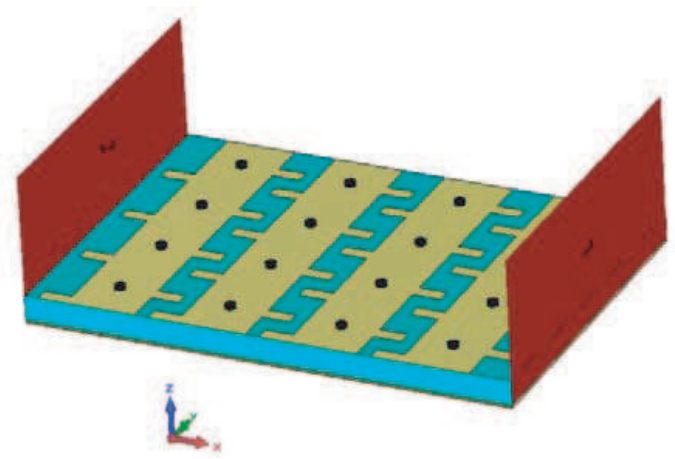

Figure 3. CST Microwave studio setup to obtain the $S$-parameters. 


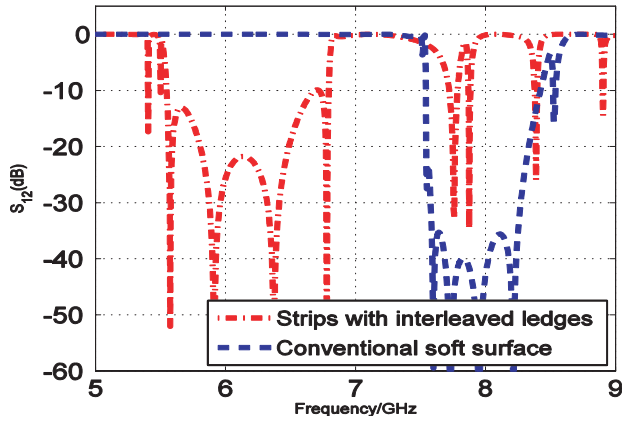

Figure 4. Comparison of the $S_{12}$ between strips with interleaved ledges and conventional planar soft surface. $N=8, w=5 \mathrm{~mm}, p_{v}=6 \mathrm{~mm}$, $p_{s}=8 \mathrm{~mm}, g=3 \mathrm{~mm}, g_{l}=0.5 \mathrm{~mm}, l=2 \mathrm{~mm}$, $w_{l}=1 \mathrm{~mm}$ and centered vias.

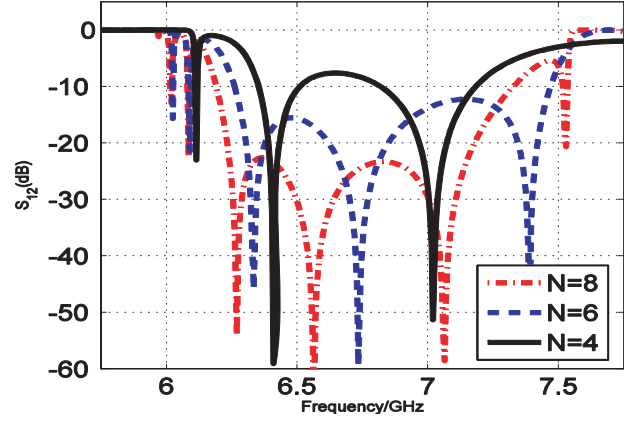

Figure 5. $S_{12}$ of different number of strips $N$. $w=5 \mathrm{~mm}, p_{v}=6 \mathrm{~mm}, p_{s}=8 \mathrm{~mm}, g=3 \mathrm{~mm}$, $g_{l}=0.5 \mathrm{~mm}, l=2 \mathrm{~mm}, w_{l}=1 \mathrm{~mm}$ and centered vias.

First, the proposed structure is compared against the planar soft surface version. For both structures $N=8, p_{v}=6 \mathrm{~mm}, w=5 \mathrm{~mm}, p_{s}=8 \mathrm{~mm}, g=3 \mathrm{~mm}$ and centered vias. For the proposed structure $g_{l}=0.5 \mathrm{~mm}, l=2.5 \mathrm{~mm}$ and $w_{l}=1 \mathrm{~mm}$. It can be concluded from Fig. 4 that the structure provides miniaturization where by the bandgap occurs at a lower frequency. The soft surface structure with interleaved ledges has a $20 \%$ relative bandgap bandwidth $\left(f_{\min }=5.55 \mathrm{GHz}, f_{\max }=6.8 \mathrm{GHz}\right.$, $f_{c}=6.18 \mathrm{GHz}$ and $\mathrm{BW}=1.25 \mathrm{GHz}$ ) whereas the conventional planar soft surface has a $10 \%$ relative bandgap bandwidth $\left(f_{\min }=7.54 \mathrm{GHz}, f_{\max }=8.33 \mathrm{GHz}, f_{c}=7.94 \mathrm{GHz}\right.$ and $\left.\mathrm{BW}=0.79 \mathrm{GHz}\right)$. The relative bandwidth is doubled. The bandgap is defined at the frequency range over which $S_{21}$ is less than $-20 \mathrm{~dB}$. It should be noted that the filtering level of the ledged structure is slightly lower than that of the conventional planar soft surface but is still below than $(-20 \mathrm{~dB})$, which provides sufficient suppression for many applications.

The effect of the number of strips $N$, the width of the strip $w$, the period $p_{v}$ and the position of the vias are examined. These parameters are important when designing soft surface structures [5]. Furthermore, the effect of the length $l$ (along the soft direction) of the ledges, ledge width $w_{l}$, and the vertical gap $g_{l}$ between interleaved ledges are also studied.

The effect of varying the number of strips $N$ on the bandgap is shown in Fig. 5 . It can be noticed that by decreasing the number of strips the filtering level also decreases from $N=8$ to $N=4$. It can also be noticed that the bandgap occurs at the same frequency range regardless of the number of strips of the interleaved ledges, which is also similar to the conventional soft surface structure [5].

The effect of the width is shown in Fig. 6 for $w=5,10$, and $15 \mathrm{~mm}$. It can be noticed that the bandgap occurs at lower frequency range when the width of the strip is larger. This is due to a larger unit cell size $p_{s}$.

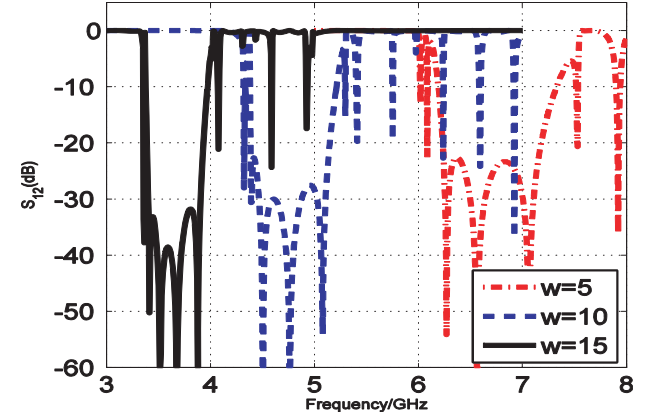

Figure 6. $S_{12}$ of different widths $w . \quad N=8$, $p_{v}=6 \mathrm{~mm}, g=3 \mathrm{~mm}, g_{l}=0.5 \mathrm{~mm}, l=2 \mathrm{~mm}$, $w_{l}=1 \mathrm{~mm}$ and centered vias.

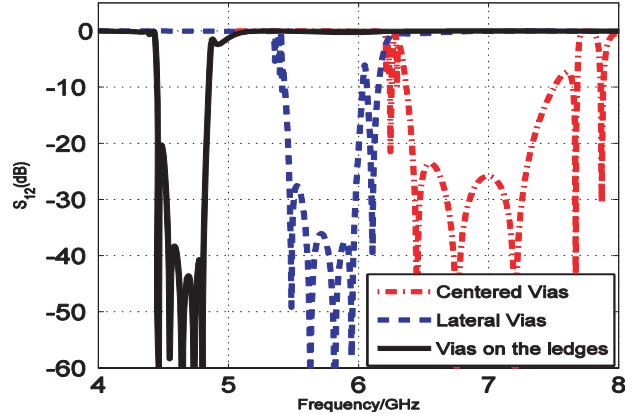

Figure 7. $S_{12}$ of different via positions. $N=8$, $w=5 \mathrm{~mm}, p_{v}=6 \mathrm{~mm}, p_{s}=8 \mathrm{~mm}, g=3 \mathrm{~mm}$, $g_{l}=1.5 \mathrm{~mm}, l=2 \mathrm{~mm}$ and $w_{l}=1 \mathrm{~mm}$. 
The results shown in Fig. 7 depicts the effect of the via position. It can be seen that the structure with the centered vias provides the widest bandgap compared with the same structure with its vias being either lateral or on the ledge. The geometries for different via positions are shown in Fig. 8. In terms of miniaturization; it is best to insert the via on the ledge. Regarding the conventional soft surface structure, the via position has the same effect in terms of miniaturization [5]. Ledges provide extra metallic area that allows adding vias hence miniaturization due to stronger capacitive coupling.

The period $p_{v}$ between vias along the strip direction (transversal to the soft direction) has an important impact on the miniaturization. This effect is clearly shown in Fig. 9 where by increasing the period $p_{v}$ to $2 p_{v}$ and $3 p_{v}\left(p_{v}=6 \mathrm{~mm}\right)$ one can obtain a miniaturized structure but with a smaller bandgap. The period $p_{v}$ has the same effect in conventional soft surfaces [5]. It is worth mentioning that the period $p_{v}$ must be less than a wavelength $\lambda_{0}$ or $\lambda_{g}$ since vias are used in the planar version of soft surfaces to replace the solid metallic wall of the corrugated version [5]. The vertical gap $g_{l}$ between the interleaved ledges is found to have no effect on the bandgap or the filtering level $S_{12}$ as seen in Fig. 10.

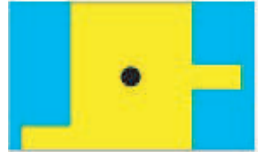

(a)

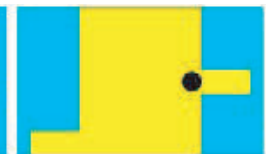

(b)

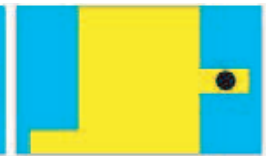

(c)

Figure 8. Geometries for different via realizations: (a) center, (b) lateral, and (c) on ledge.

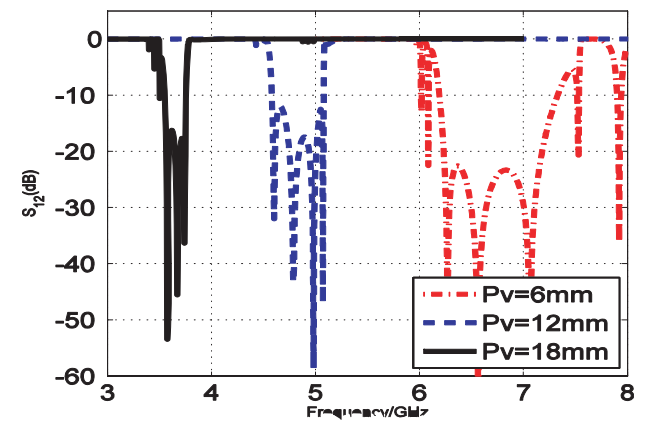

Figure 9. $S_{12}$ of different periods $p_{v} . \quad N=8$, $w=5 \mathrm{~mm}, p_{s}=8 \mathrm{~mm}, g=3 \mathrm{~mm}, g_{l}=0.5 \mathrm{~mm}$, $l=2 \mathrm{~mm}, w_{l}=1 \mathrm{~mm}$ and centered vias.

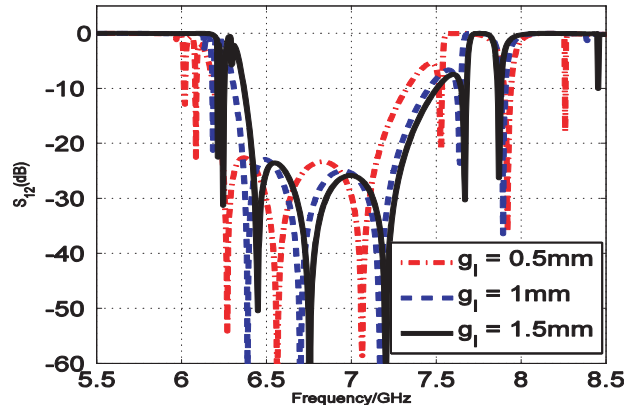

Figure 10. $S_{12}$ of different vertical gaps $g_{l}$. $N=8, w=5 \mathrm{~mm}, p_{v}=6 \mathrm{~mm}, p_{s}=8 \mathrm{~mm}$, $g=3 \mathrm{~mm}, l=2 \mathrm{~mm}, w_{l}=1 \mathrm{~mm}$ and centered vias.

A miniaturized structure can be obtained by increasing the length $l$ of the ledge along the soft direction provided that it should not touch the next strip and maintaining the same actual gap $g$ between the strips, hence the same cell size. The filtering level gets slightly lower with $l$ being increased, but it is still below $-20 \mathrm{~dB}$. Moreover, by increasing the ledge length the bandgap value is not affected. The effect of $l$ is shown in Fig. 11. Finally, a slightly miniaturized structure can be obtained also by increasing the ledge width $w_{l}$ as shown in Fig. 12. The ledge width does not affect the filtering level as shown in Fig. 12.

Table 1 summarizes the main conclusions obtained from the considered parametric study and the corresponding relative bandgap bandwidth. To design a soft surface that has a $20 \%$ relative bandgap bandwidth with a suppression level of $-20 \mathrm{~dB}$; one can set: $l=2.5 \mathrm{~mm}, N=8, w=5 \mathrm{~mm}, p_{v}=6 \mathrm{~mm}$, $p_{s}=8 \mathrm{~mm}, g=3 \mathrm{~mm}, g_{l}=0.5 \mathrm{~mm}, w_{l}=1 \mathrm{~mm}$ and centered vias. 


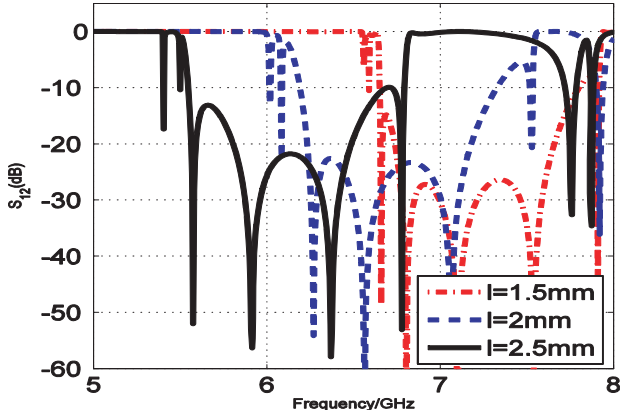

Figure 11. $S_{12}$ of different ledge lengths $l . N=$ $8, w=5 \mathrm{~mm}, p_{v}=6 \mathrm{~mm}, p_{s}=8 \mathrm{~mm}, g=3 \mathrm{~mm}$, $g_{l}=0.5 \mathrm{~mm}, w_{l}=1 \mathrm{~mm}$ and centered vias.

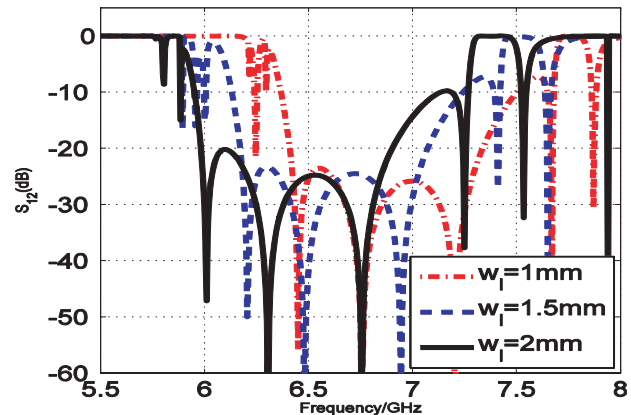

Figure 12. $S_{12}$ of different ledge widths $w_{l}$. $N=8, w=5 \mathrm{~mm}, p_{v}=6 \mathrm{~mm}, p_{s}=8 \mathrm{~mm}$, $g=3 \mathrm{~mm}, g_{l}=1.5 \mathrm{~mm}, l=2 \mathrm{~mm}$ and centered vias.

Table 1. Percent BW of different parameters.

\begin{tabular}{|c|c|}
\hline Parameter & BW $(\%)$ at $-20 \mathrm{~dB}$ \\
\hline$N=4$ strips & $10 \%$ \\
$N=6$ strips & $12 \%$ \\
$N=8$ strips & $14 \%$ \\
\hline$w=5 \mathrm{~mm}$ & $14 \%$ \\
$w=10 \mathrm{~mm}$ & $16 \%$ \\
$w=15 \mathrm{~mm}$ & $15 \%$ \\
\hline Via (center) & $13.45 \%$ \\
Via (lateral) & $9.44 \%$ \\
Via (ledge) & $8.1 \%$ \\
\hline$p_{v}=6 \mathrm{~mm}$ & $14 \%$ \\
$p_{v}=12 \mathrm{~mm}\left(2 p_{v}\right)$ & $10.19 \%$ \\
$p_{v}=18 \mathrm{~mm}\left(3 p_{v}\right)$ & $5.01 \%$ \\
\hline$g_{l}=0.5 \mathrm{~mm}$ & $14 \%$ \\
$g_{l}=1 \mathrm{~mm}$ & $13.9 \%$ \\
$g_{l}=1.5 \mathrm{~mm}$ & $13.55 \%$ \\
\hline$l=1.5 \mathrm{~mm}$ & $14.05 \%$ \\
$l=2 \mathrm{~mm}$ & $14 \%$ \\
$l=2.5 \mathrm{~mm}$ & $19.8 \%$ \\
\hline$w_{l}=1 \mathrm{~mm}$ & $13.54 \%$ \\
$w_{l}=1.5 \mathrm{~mm}$ & $13.61 \%$ \\
$w_{l}=2 \mathrm{~mm}$ & $14.29 \%$ \\
\hline
\end{tabular}

\section{EXPERIMENTAL RESULTS}

\subsection{Mutual Coupling between Two Monopoles}

In this section, the same experimental approach in [4] is used to characterize the soft surface bandgap where two quarterwave vertical monopoles are used to measure coupling for vertical polarizations; each have $10.5 \mathrm{~mm}$ in height above the substrate. The two monopoles resonate at about $f_{0}=7.15 \mathrm{GHz}$. Fig. 13 shows a photo of the fabricated prototype. The two monopoles are surrounded by a soft 
surface structure. Monopoles are inserted in the middle between strips two and three and between strips six and seven and soldered with SMA connectors as shown in Figs. 13(a) and (b). Four periods are used along the length of the strips. For this prototype; $N=8, w=5 \mathrm{~mm}, p_{v}=6 \mathrm{~mm}, p_{s}=8 \mathrm{~mm}$, $g=3 \mathrm{~mm}, g_{l}=0.5 \mathrm{~mm}, l=2 \mathrm{~mm}, w_{l}=1 \mathrm{~mm}$ and centered vias are used. The grounded dielectric slab is Rogers RO3003 with a relative permittivity $\varepsilon_{r}=3$, loss tangent $\tan \delta=0.0013$, and thickness $=1.524 \mathrm{~mm}$. The two ports of the prototype have been connected to a vector network analyzer and the $S_{12}$ parameter is measured. N5242A PNA-X Microwave Network Analyzer from Agilent Technologies is used for measurements. The Network Analyzer is calibrated for 1001 points from $6 \mathrm{GHz}$ to $8 \mathrm{GHz}$. The prototype shown in Fig. 13 is simulated by modeling the monopoles and including the losses of the dielectric slab and metals. Computed and measured mutual coupling between the ports of the two monopoles are shown in Fig. 14. Also, the two monopoles are simulated and manufactured over a conducting plane (hard surface for the monopole polarization) to compare mutual coupling against the soft surface structure using interleaved ledges. The coupling at $7.15 \mathrm{GHz}$ has been reduced using the soft surface structure with interleaved ledges from $-15 \mathrm{~dB}$ to $-44 \mathrm{~dB}$.

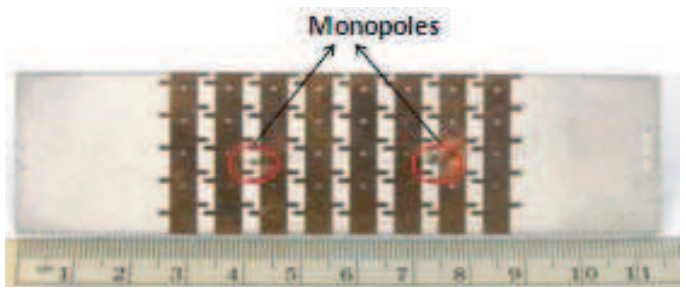

(a) Top view

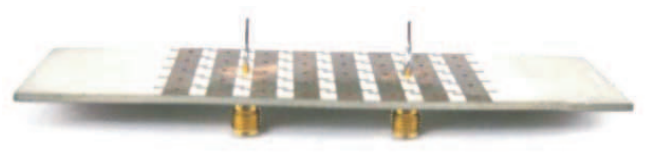

(b) Tilted view

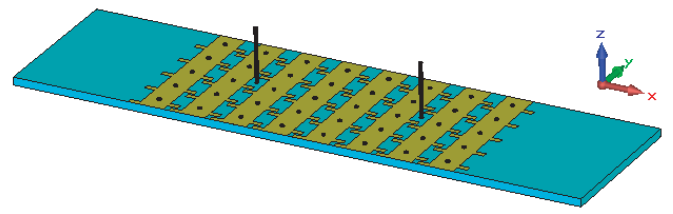

(c) Simulated prototype model using CST

Figure 13. Photos of the prototype and the two monopoles surrounded by soft surface.

\subsection{Mutual Coupling between Two Patch Antennas}

Planar soft surfaces are promising when it comes to mutual coupling reduction between patch antennas. Two patch antennas are designed to provide enough coupling. An important issue when adding the soft surface between two radiating patch antennas is to avoid the potential effects of such structures on the radiation patterns; for instance such an issue has not been reported in [16-18]. Finally, a large distance between the two patch antennas $0.6 \lambda_{0}$ and $0.76 \lambda_{0}$ are taken in $[5,14]$, respectively, which indeed not allow for sufficient coupling since $S_{21}$ is already below $-20 \mathrm{~dB}$ at the resonant frequency.

Two patch antennas are designed to resonate at $5.8 \mathrm{GHz}$. The substrate is RO4835 with a dielectric constant $\varepsilon_{r}=3.48$ and thickness $t=1.52 \mathrm{~mm}$. A unit cell with $p_{s}=6.8 \mathrm{~mm}$ has been used. Moreover, $p_{v}=6 \mathrm{~mm}$ (with 7 periods along the length of the strips), strip width $w=3.8 \mathrm{~mm}, g=3 \mathrm{~mm}$, length of ledge $l=2.5 \mathrm{~mm}, w_{l}=1 \mathrm{~mm}$, vertical gap between interleaved ledges $g_{l}=2 \mathrm{~mm}$ and the via position is on the ledge of the left strip. The soft surface parameters were chosen so that the structure provides a bandgap at $5.8 \mathrm{GHz}$. The distance (center to center) between the two patch antennas is $25 \mathrm{~mm}$ which is slightly less than $0.5 \lambda_{0}$ at $5.8 \mathrm{GHz}$. The proposed structure is shown in Fig. 15.

Before adding the soft surface, the rectangular patches have dimensions of $12.5 \times 15.7 \mathrm{~mm}^{2}$. The feed position is $3.85 \mathrm{~mm}$ from the edge for the two patches. By adding the soft surface as shown in Fig. 15, the size of the two patch antennas are slightly adjusted to $12.7 \times 15.7 \mathrm{~mm}^{2}$ and the feed position also changed to $3.65 \mathrm{~mm}$ from the edge for the two patches. The substrate dimensions are $67 \times 42 \mathrm{~mm}^{2}$ $\left(1.3 \lambda_{0} \times 0.81 \lambda_{0}\right)$. Simulation results are shown in Fig. 16. The mutual coupling $S_{21}$ at $5.8 \mathrm{GHz}$ without

the soft surface is $-18 \mathrm{~dB}$ whereas it decreased to $-28 \mathrm{~dB}$ when using the soft surface. The matching 


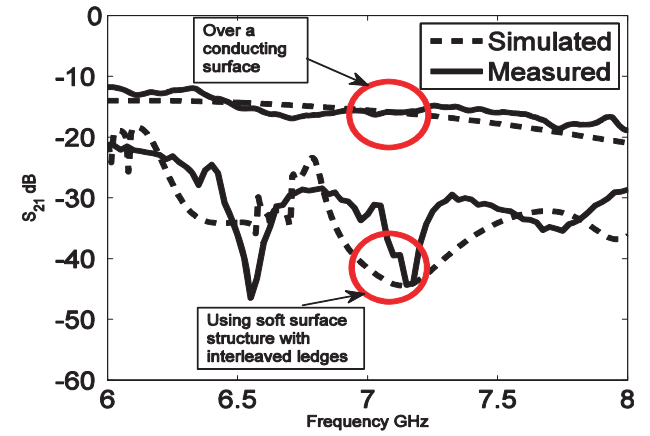

Figure 14. Measured and computed mutual coupling between the two monopoles over a conducting surface and the soft surface for the prototype in Fig. 13.

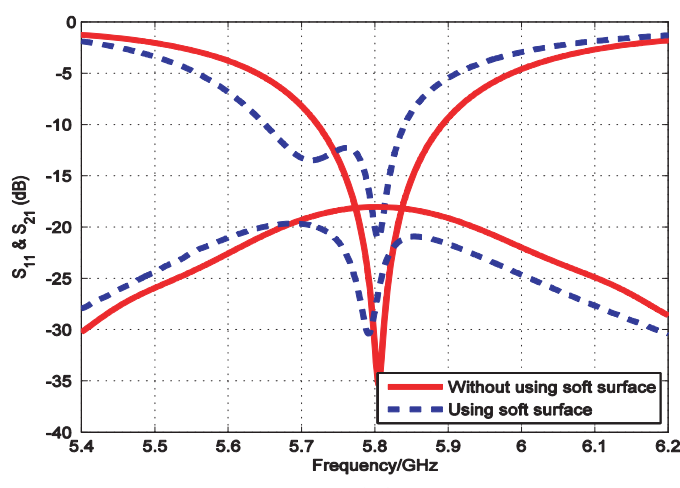

(a)

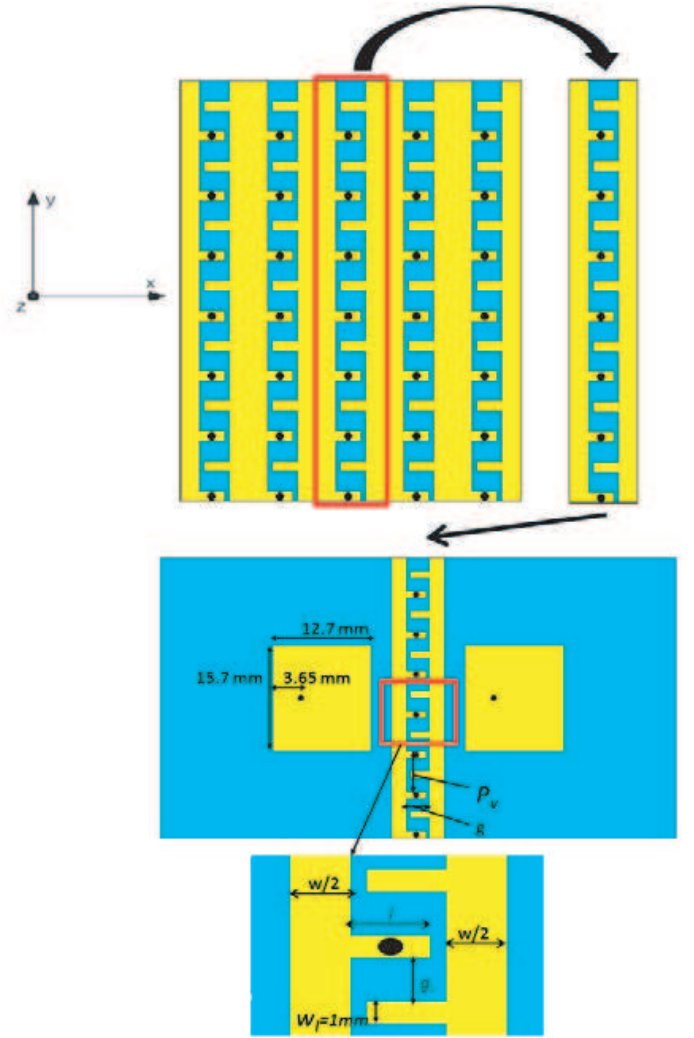

Figure 15. Top view of patch antenna array decoupled by one unit cell of soft surface structure of interleaved ledges.

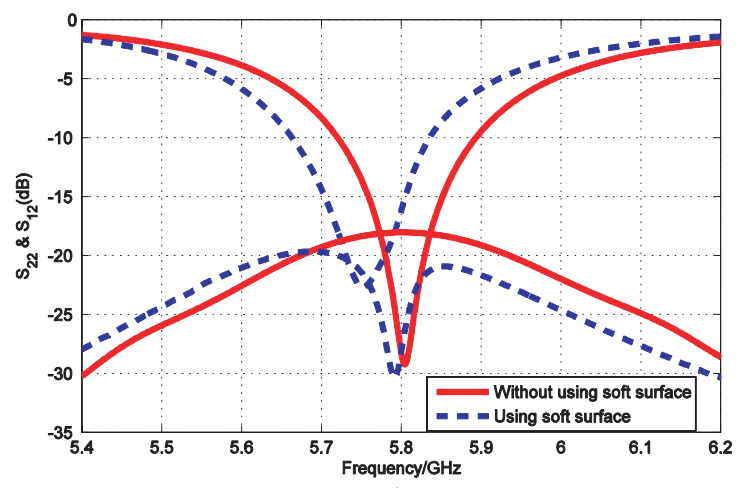

(b)

Figure 16. $S$-parameters for the two patches in Fig. 15 with and without the soft surface structure. (a) $S_{11}$ and $S_{21}$ for the left patch, (b) $S_{22}$ and $S_{12}$ for the right patch.

bandwidth for reflection coefficients below $-10 \mathrm{~dB}$ is $0.19 \mathrm{GHz}(5.65-5.84 \mathrm{GHz})$, which is equivalent to $3.3 \%$ whereas it is $0.18 \mathrm{GHz}(5.72-5.9 \mathrm{GHz})$, which is equivalent to $3.1 \%$ for the array without the soft surface. The modified patch size and feed position could keep almost the same bandwidth but with a slight shift in resonant frequency. The reduction in mutual coupling occurs over a $2 \% \mathrm{BW}$, from $5.72 \mathrm{GHz}$ to $5.84 \mathrm{GHz}$.

By simultaneously exciting the two ports of the antennas as in Fig. 15 a slight increase in back 


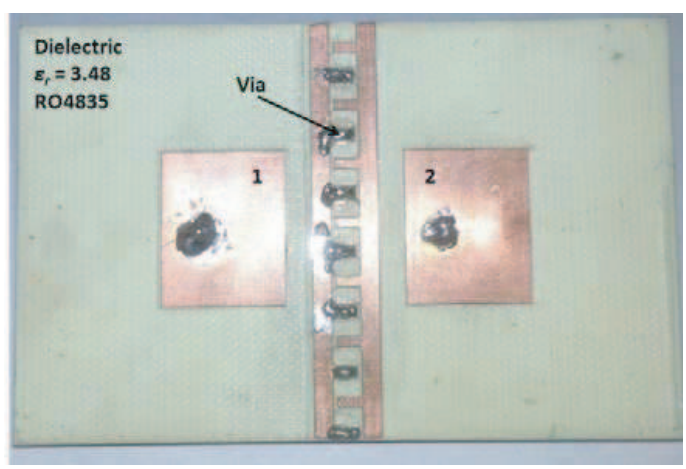

Figure 17. Prototype of the proposed structure in Fig. 15.
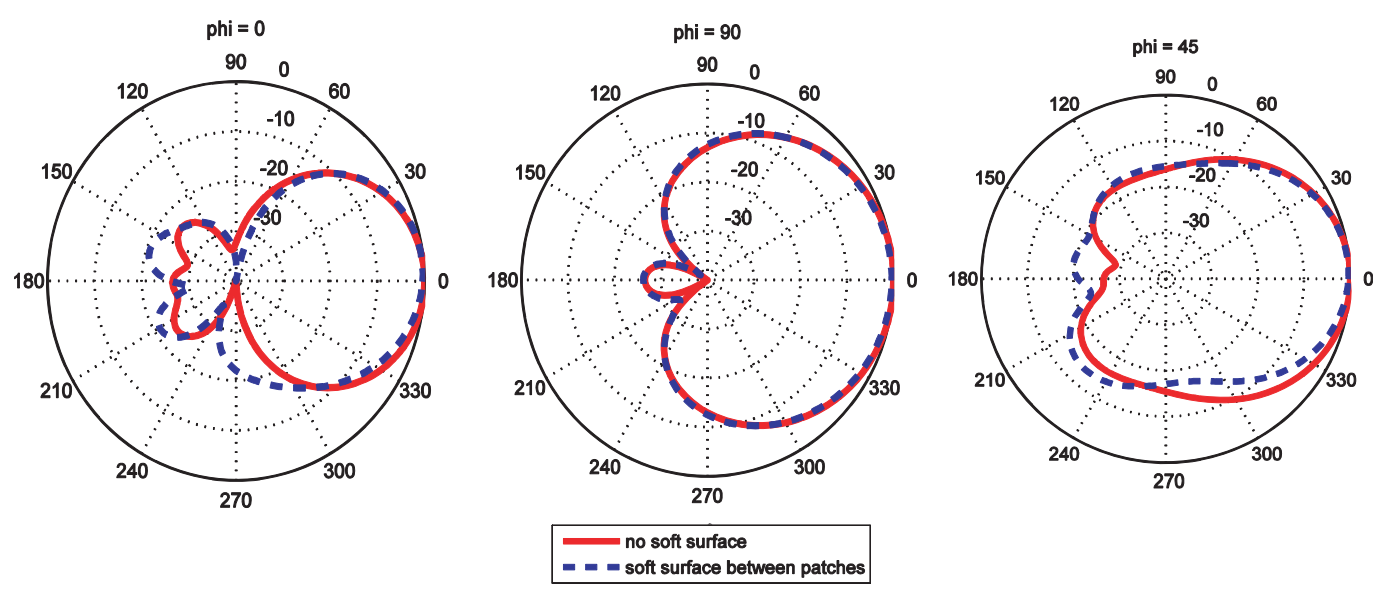

Figure 18. Radiation patterns for the two patches in different planes with and without the soft surface between the patches.

radiation is observed compared with the original model (without soft surface). Simulation results for the three plane cuts $\varphi=0^{\circ}, \varphi=90^{\circ}, \varphi=45^{\circ}$ are shown in Fig. 18, it is noticed that the radiation patterns did not posses asymmetric in any plane by adding the soft surface structure of interleaved ledges. By adding conventional soft surface structure, it was found in [5] that the radiation pattern shows asymmetrical characteristics particularly in the $E$-plane $(\varphi=0)$. To resolve this issue in the conventional soft surface structures, the strips are mirrored around the patches, which requires even number of strips. As such, extra space between the patches is needed, which is not required in the present design.

Table 2 shows a comparison between the proposed design decoupled by a planar soft surface with interleaved ledges and other different techniques for decoupling mentioned in the literature to reduce the mutual coupling between E-coupled microstrip patch antenna elements. The radiation pattern did not get that much disturbance by adding the soft surface structure of interleaved ledges. Fig. 19 shows the embedded radiation patterns for the two patches separately. Patch 1 is on the left in Fig. 15. When the mutual coupling is high, the radiation patterns in the $E$-plane $(\varphi=0)$ and $(\varphi=45)$ cuts are not in the boresight direction whereas it is in the boresight after reducing the mutual coupling. The main benefit of the soft surface occurs by decreasing the mutual coupling between the antennas making the radiation pattern in the boresight. Tables 3,4 , and 5 show the values of the main lobe magnitude $(\mathrm{dB})$, main lobe direction (degrees), angular width (3 dB) (degrees) and the side lobe level $(\mathrm{dB})$ for the three cases: simultaneous excitation of the two patches, patch element 1 and patch element 2, all at 5.8 GHz. Results for the antenna array without using the soft surface structure of interleaved ledges, and by using the soft surface structure of interleaved ledges for mutual coupling reduction are compared together.

The proposed structure in Fig. 15 was fabricated as shown in Fig. 17. The $S$-parameters are 
Table 2. Performance of the proposed interleaved ledges and other geometries in literature for $E$-plane decoupling in microstrip patch antenna array.

\begin{tabular}{|c|c|c|c|c|c|c|c|c|}
\hline No. & $\begin{array}{l}\text { Ref. } \\
\text { No. }\end{array}$ & Technique & $\begin{array}{c}\text { Size of the } \\
\text { array in } \mathrm{mm}^{2}\end{array}$ & $\begin{array}{c}\text { Frequency } \\
\left(f_{0}\right) \text { in } \mathrm{GHz}\end{array}$ & $\begin{array}{c}-10 d B \\
\text { Percentage } \\
\text { Bandwidth }\end{array}$ & $\begin{array}{l}\text { Edge to } \\
\text { Edge } \\
\text { Spacing }\end{array}$ & $\begin{array}{c}\text { Center to } \\
\text { Center } \\
\text { Spacing }\end{array}$ & $\begin{array}{l}\text { Improvement } \\
\text { in } S_{21}(d B)\end{array}$ \\
\hline 1 & {$[18]$} & $\begin{array}{c}\text { Multilayer Dielectric } \\
\text { Substrate + EBG }\end{array}$ & $130 \times 130$ & 3.0 & 7.67 & $\begin{array}{c}0.40 \lambda_{0} \\
(40 \mathrm{~mm})\end{array}$ & $\begin{array}{c}0.75 \lambda_{0} \\
(75 \mathrm{~mm})\end{array}$ & 10.00 \\
\hline 2 & {$[19]$} & $\begin{array}{c}\text { Uniplanar EBG } \\
\text { over Superstrate }\end{array}$ & $78.3 \times 78.3$ & 5.75 & 1.74 & $\begin{array}{c}0.50 \lambda_{0} \\
(26.0 \mathrm{~mm})\end{array}$ & $\begin{array}{c}0.63 \lambda_{0} \\
(32.9 \mathrm{~mm})\end{array}$ & 10.00 \\
\hline 3 & {$[20]$} & $\begin{array}{c}\text { Mushroom-type } \\
\text { EBG }\end{array}$ & $100.0 \times 50.0$ & 5.8 & Not-reported & $\begin{array}{c}0.75 \lambda_{0} \\
(38.8 \mathrm{~mm}) \\
\end{array}$ & $\begin{array}{c}0.88 \lambda_{0} \\
(45.6 \mathrm{~mm})\end{array}$ & 8.00 \\
\hline 4 & {$[14]$} & $\begin{array}{c}\text { Fork-like } \\
\text { EBG }\end{array}$ & Not-reported & 5.2 & Not-reported & $\begin{array}{c}0.73 \lambda_{0} \\
(38 \mathrm{~mm})\end{array}$ & $\begin{array}{c}0.76 \lambda_{0} \\
(44 \mathrm{~mm})\end{array}$ & 6.51 \\
\hline 5 & {$[21]$} & DGS & Not-reported & 10.0 & 11.2 & $\begin{array}{c}0.70 \lambda_{0} \\
(21 \mathrm{~mm})\end{array}$ & $\begin{array}{c}0.9 \lambda_{0} \\
(27.13 \mathrm{~mm})\end{array}$ & 9.00 \\
\hline 6 & {$[22]$} & Slotted CSRR & $78.0 \times 60.0$ & 5.0 & 3.00 & $\begin{array}{c}0.25 \lambda_{0} \\
(15 \mathrm{~mm})\end{array}$ & $\begin{array}{c}0.5 \lambda_{0} \\
(30.0 \mathrm{~mm})\end{array}$ & 10.00 \\
\hline 7 & {$[5]$} & $\begin{array}{c}\text { Conventional } \\
\text { planar soft } \\
\text { surface }\end{array}$ & Not-reported & 1.92 & Not-reported & $\begin{array}{c}0.36 \lambda_{0} \\
(57 \mathrm{~mm})\end{array}$ & $\begin{array}{c}0.6 \lambda_{0} \\
(93 \mathrm{~mm})\end{array}$ & 10.00 \\
\hline 8 & $\begin{array}{c}\text { (proposed } \\
\text { design) }\end{array}$ & $\begin{array}{c}\text { Interleaved } \\
\text { planar soft } \\
\text { surface }\end{array}$ & $67.0 \times 42.0$ & 5.8 & 3.3 & $\begin{array}{c}0.23 \lambda_{0} \\
(12.3 \mathrm{~mm})\end{array}$ & $\begin{array}{c}0.48 \lambda_{0} \\
(25 \mathrm{~mm})\end{array}$ & 13.00 \\
\hline
\end{tabular}

Table 3. Simultaneous excitation results.

\begin{tabular}{|c|c|c|c|c|c|c|}
\hline & \multicolumn{3}{|c|}{ No soft surface } & \multicolumn{2}{c|}{ Soft surface between patches } \\
\hline$\varphi$ & 0 & 90 & 45 & 0 & 90 & 45 \\
\hline main lobe magnitude & 9.2 & 9.2 & 9.2 & 8.9 & 8.8 & 8.9 \\
\hline main lobe direction & 0 & 0 & 0 & 4 & 1 & 4 \\
\hline angular width (3dB) & 49.6 & 79.7 & 60.8 & 47.7 & 81.4 & 52.9 \\
\hline side lobe level & -24.1 & -26.5 & -16.2 & -20.4 & -25.8 & -13.3 \\
\hline
\end{tabular}

Table 4. Patch element 1 excitation results.

\begin{tabular}{|c|c|c|c|c|c|c|}
\hline & \multicolumn{3}{|c|}{ No soft surface } & \multicolumn{2}{c|}{ Soft surface between patches } \\
\hline$\varphi$ & 0 & 90 & 45 & 0 & 90 & 45 \\
\hline main lobe magnitude & 7.6 & 6.4 & 7.1 & 4.8 & 4.8 & 5.1 \\
\hline main lobe direction & 18 & 0 & 16 & 1 & 2 & 0 \\
\hline angular width (3dB) & 65 & 77.8 & 72.8 & 48.9 & 93.9 & 50.2 \\
\hline side lobe level & -17.1 & -24.4 & -17.1 & -11.1 & -17 & -2.8 \\
\hline
\end{tabular}

Table 5. Patch element 2 excitation results.

\begin{tabular}{|c|c|c|c|c|c|c|}
\hline & \multicolumn{3}{|c|}{ No soft surface } & \multicolumn{2}{c|}{ Soft surface between patches } \\
\hline$\varphi$ & 0 & 90 & 45 & 0 & 90 & 45 \\
\hline main lobe magnitude & 7.6 & 6 & 7 & 7.1 & 6.8 & 7.1 \\
\hline main lobe direction & 19 & 0 & 18 & 10 & 1 & 12 \\
\hline angular width (3dB) & 58 & 81.8 & 69.8 & 75.8 & 76.8 & 101.3 \\
\hline side lobe level & -3.4 & -26.8 & -18.5 & -32.2 & -27.2 & -11.9 \\
\hline
\end{tabular}



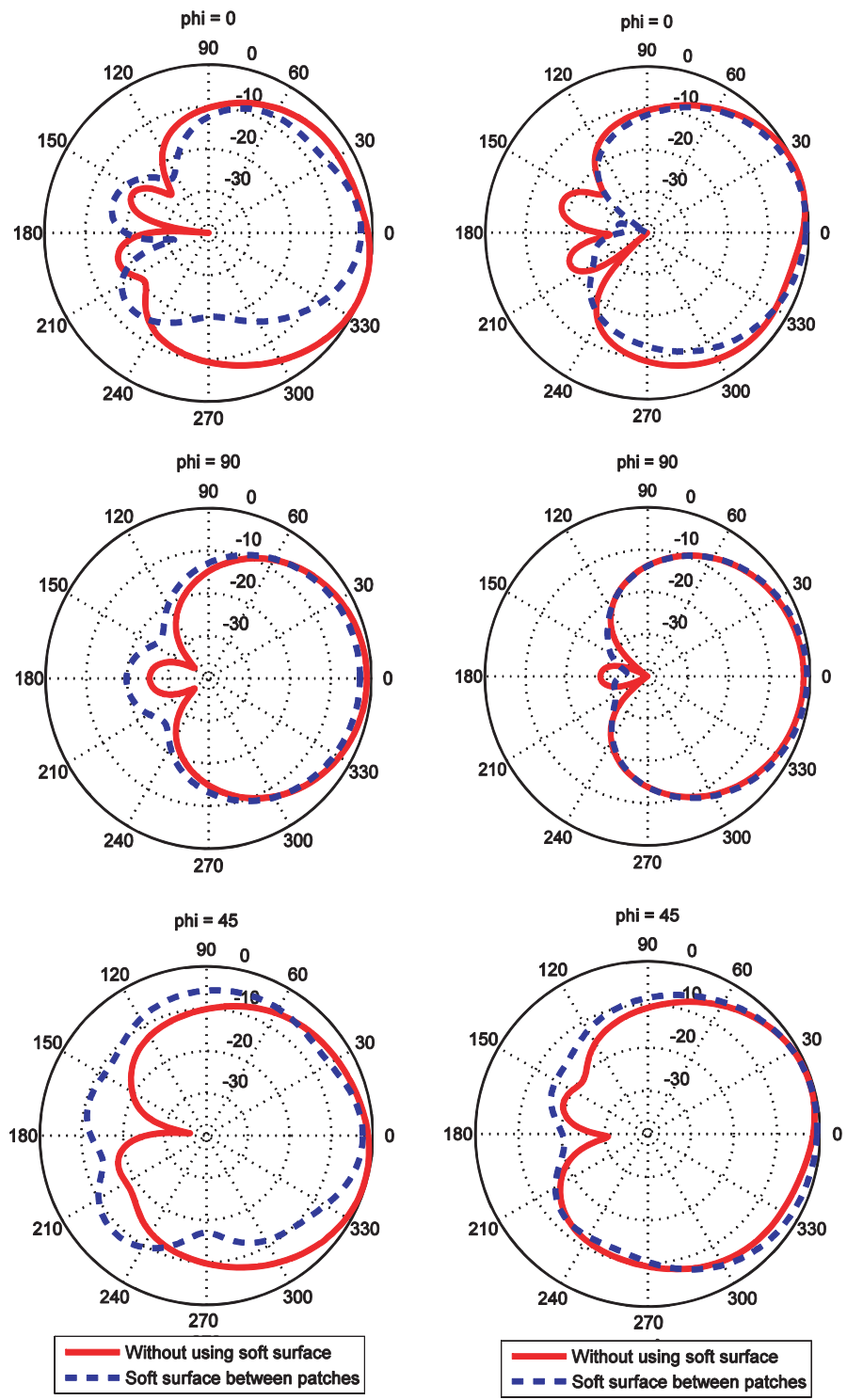

Figure 19. Embedded radiation patterns for the two elements. Left side for patch 1 and right side for patch 2 .

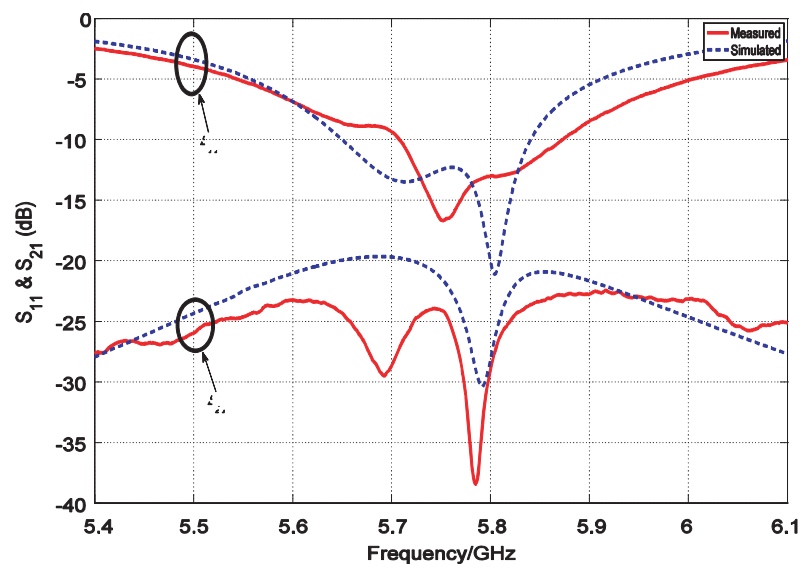

Figure 20. Measured versus simulated $S_{11}$ and $S_{21}$. 


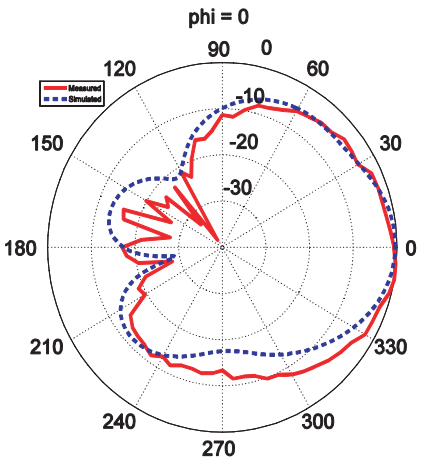

(a) E-plane

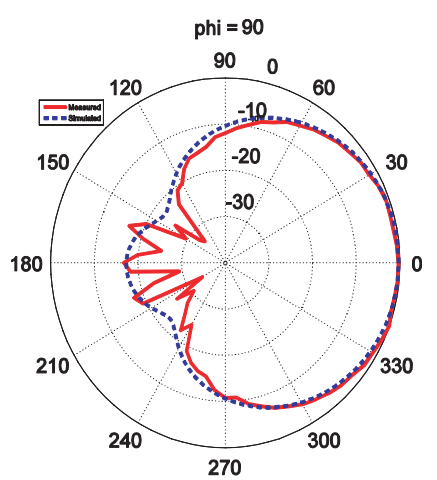

(b) $H$-plane

Figure 21. Measured versus simulated far-field characteristics of antenna element 1 in patch antenna array. (a) E-plane pattern. (b) $H$-plane pattern.

measured using the same network analyzer mentioned in the previous section. The network analyzer was calibrated from $5.4 \mathrm{GHz}$ to $6.1 \mathrm{GHz}$ for 1001 points. Measured $S$-parameters were compared with the simulated ones and they show enough agreement as shown in Fig. 20. Moreover, the radiation pattern in the $E$-plane and $H$-plane for patch 1 is shown in Fig. 21. Patch 2 was terminated by $50-\Omega$ load.

\section{CONCLUSION}

A new version of a planar soft surface is designed, simulated and measured. By changing different parameters such as the number of strips $N$, period between vias $p_{v}$, width of strip $w$, length of ledge $l$, width of ledge $w_{l}$, vertical gap between interleaved ledges $g_{l}$ and position of vias, different designs were obtained and characterized. The length of the ledge $l$ which is along the soft direction is a very important parameter. It provided a $20 \%$ relative bandwidth of the bandgap whereas the conventional planar soft surface structure gave a relative bandwidth of $10 \%$. A more compact structure was obtained by inserting these vias on the ledges compared with the lateral via position. The new soft surface structure was used efficiently to reduce the mutual coupling in $2 \times 1$ microstrip patch antenna array without affecting the bandwidth.

\section{REFERENCES}

1. Kildal, P., "Definition of artificially soft and hard surfaces for electromagnetic waves," Electronics Letters, Vol. 24, No. 3, 168-170, 1988.

2. Kildal, P., "Artificially soft and hard surfaces in electromagnetics," IEEE Trans. Antennas Propag., Vol. 38, No. 10, 1537-1544, 1990.

3. Kildal, P., "Artificially soft and hard surfaces in electromagnetics and their application to antenna design," 23rd European Microwave Conference, 1993, 30-33, Madrid, Spain, Sep. 6-10, 1993.

4. Kildal, P. and A. Kishk, "EM modeling of surfaces with stop or go characteristics - Artificial magnetic conductors and soft and hard surfaces," ACES Journal, Vol. 18, No. 1, 32-40, 2003.

5. Rajo-Iglesias, E., Ó. Quevedo-Teruel, and L. Inclán-Sánchez, "Planar soft surfaces and their application to mutual coupling reduction," IEEE Trans. Antennas Propag., Vol. 57, No. 12, 38523859, 2009.

6. Sciré-Scappuzzo, F. and S. N. Makarov, "A low-multipath wideband GPS antenna with cutoff or non-cutoff corrugated ground plane," IEEE Trans. Antennas Propag., Vol. 57, No. 1, 33-46, 2009.

7. Simon, A. E. and A. A. Kishk, "Asymptotic strip boundary condition in the finite difference time domain method," IEEE Trans. Antennas Propag., Vol. 53, No. 3, 1187-1193, Mar. 2005. 
8. Simon, A. E. and A. A. Kishk, "Asymptotic corrugated boundary condition in the finite difference time domain method," Radio Science, Vol. 40, RS6S05, 8, 2005, doi: 10.1029/2004RS003148.

9. Rajo-Iglesias, E., L. Inclán-Sánchez, and Ó. Quevedo-Teruel, "Back radiation reduction in patch antennas using planar soft surfaces," Progress In Electromagnetics Research Letters, Vol. 6, 123$130,2009$.

10. Sievenpiper, D., L. Zhang, R. F. Jimenez Broas, N. Alexópolous, and E. Yablonovitch, "Highimpedance electromagnetic surfaces with a forbidden frequency band," IEEE Trans. Microw. Theory Tech., Vol. 47, No. 11, 2059-2074, 1999.

11. Rajo-Iglesias, E., M. Caiazzo, L. Inclan-Sanchez, and P. Kildal, "Comparison of bandgaps of mushroom-type EBG surface and corrugated and strip-type soft surfaces," Microwaves, Antennas \& Propagation, IET, Vol. 1, No. 1, 184-189, 2007.

12. Thai, T., G. DeJean, and M. Tentzeris, "Design and development of a novel compact soft-surface for the front-to-back ratio improvement and size reduction of a microstrip yagi array antenna," IEEE Trans. Antennas Propag. Lett., Vol. 7, 369-373, 2008.

13. Li, R., G. DeJean, M. M. Tentzeris, J. Papapolymerou, and J. Laskar, "Radiation-pattern improvement of patch antennas on a large-size substrate using a compact soft-surface structure and its realization on LTCC multilayer technology," IEEE Trans. Antennas Propag., Vol. 53, No. 1, 200-208, 2005.

14. Yang, L., M. Fan, F. Chen, J. She, and Z. Feng, "A novel compact electromagnetic-bandgap (EBG) structure and its applications for microwave circuits," IEEE Trans. Microw. Theory Tech., Vol. 53, No. 1, 183-190, 2005.

15. Abushamleh, S., H. Al-Rizzo, A. Kishk, and H. Khaleel, "Enhancement of the strips electromagnetic soft surfaces using ledge edges," Antennas and Propagation Society International Symposium (APSURSI), 2012 IEEE, 1-2, July 2012.

16. Zheng, Q. R., Y. O. Fu, and N. C. Yuan, "A novel compact spiral electromagnetic band-gap (EBG) structure," IEEE Trans. Antennas Propag., Vol. 56, 1656-1660, Jun. 2008.

17. Chiu, C. Y., C. H. Cheng, R. Murch, and C. Rowell, "Reduction of mutual coupling between closely-packed antenna elements," IEEE Trans. Antennas Propag., Vol. 55, 1732-1738, Jun. 2007.

18. Rajo-Iglesias, E., Ó. Quevedo-Teruel, and L. Inclán-Sánchez, "Mutual coupling reduction in patch antenna arrays by using a planar periodic structure and a multilayer dielectric substrate," IEEE Trans. Antennas Propag., Vol. 56, 1648-1655, Jun. 2008.

19. Farahani, H. S., M. Veysi, M. Kamyab, and A. Tadjalli, "Mutual coupling reduction in patch antenna arrays using a UC-EBG superstrate," IEEE Trans. Antennas Wireless Propag. Lett., Vol. 9, 57-59, 2010.

20. Yang, F. and Y. Rahmat-Samii, "Microstrip antennas integrated with electromagnetic band-gap (EBG) structures: A low mutual coupling design for array applications," IEEE Trans. Antennas Propag., Vol. 51, No. 10, 2936-2946, Oct. 2003.

21. Vazquez-Antuna, C., G. R. Hotopan, S. Ver-Hoeye, M. Fernandez-Garcia, L. F. Herran Ontanon, and F. Las Heras Andres, "Defected ground structure for coupling reduction between probe fed microstrip antenna elements," PIERS Proceedings, 640-644, Cambridge, USA, Jul. 5-8, 2010.

22. Bait-Suwailam, M. M., O. F. Siddiqui, and O. M. Ramahi, "Mutual coupling reduction between microstrip patch antennas using slotted-complementary split-ring resonators," IEEE Antennas Wireless Propag. Lett., Vol. 9, 876-878, 2010. 